\title{
FOBIA A CONTRAER LINFEDEMA: CASO CLÍNICO
}

\author{
PHOBIA TO CONTRACT LYMPHEDEMA: CLINICAL CASE
}

\author{
Lorena Alonso Rivas' y M Helena López de Ceballos Reyna ${ }^{2}$ \\ ' Junta Provincial de Cáceres de la Asociación Española Contra el cáncer \\ 2 Hospital San Pedro de Alcántara. Cáceres
}

Resumen

Introducción: análisis de un caso clínico de mujer operada de mama con linfadenectomía remitida por su oncólogo en el año 2009 al no realizar ningún movimiento con el brazo por temor a contraer linfedema.

Objetivos: 1. Psicoeducación sobre el linfedema y cuidados del brazo. 2. Vencer el miedo al movimiento del brazo. 3. Mejorar la calidad de vida de la paciente.

Metodología: eelaboración de un programa de exposición a través de las situaciones en que la paciente experimentaba gran ansiedad y temor, relacionadas con actividades habituales de su vida diaria, realizando la siguiente lista de actividades, de menor a mayor ansiedad:comer, vestirse, labores domésticas, aseo personal y realizar ejercicio físico. Se utilizó el autorregistro con las tareas de exposición realizadas.

Resultados: la paciente superó sus miedos hacia el linfedema, realizando una vida totalmente normal, conociendo cuáles son los cuidados que debe tener a modo de prevención.

Conclusiones: el tratamiento de exposición ha mostrado eficacia en el presente caso y confirma que es una intervención en el caso de fobias en pacientes oncológicos.

Palabras clave: Fobia, linfedema, exposición, cáncer mama, psicoeducación.

\section{Abstract}

Introduction: Clinical case study of a woman who has been operated on breast cancer and lymphadenectomy who was referred by her oncologist in 2009, given because she didn't move her arm for fear of acquire lymphedema.

Purposes: 1. Psychoeducation about lymphedema and how to look after the arm. 2. Overcoming fear of moving the arm. 3 . Improving the patient's quality of life.

Method: Designing an exposure therapy in which the patient has to cope with situations that arouse great anxiety and fear. These situations are related to common activities in the patient's daily life and has been rated in a list of increasing level of anxiety: Eating, getting dressed, housework, personal hygiene and physical exercise.

The patient kept a record book with the exposure tasks that she confronted.

Results: The patient overcame all her fears for lymphedema, leading a completely normal life and learning about the measures and cares to take for lymphedema prevention.

Conclusions: Exposure is the most appropriate treatment option for phobic disorders in patients who suffer cancer.

Keywords: Phobia, lymphedema, exposure, breast cancer, psychoeducation.

Correspondencia:

Lorena Alonso Rivas

C/Donantes de Sangre 1

10002-Cáceres

E-mail: lorena.alonso@aecc.es 


\section{INTRODUCCIÓN}

El diagnóstico de cáncer con frecuencia suele suponer un gran impacto en el paciente, presentándose en él sintomatología ansiosa depresiva en un primer momento, siendo reacciones adaptativas a la situación vivida. A continuación, la mayor parte de los pacientes son capaces de enfrentarse de forma adaptativa a la enfermedad, sus efectos y los cambios de vida que esto supone, solos o con la ayuda de familiares y personal sanitario, sin que lleguen a padecer ningún trastorno mental. Sin embargo, hay un porcentaje de pacientes de cáncer que desarrollan cierta patología debido a una vulnerabilidad previa, la presencia de otros acontecimientos estresantes, la ausencia de estrategias de afrontamiento adecuadas o un déficit en apoyo social, así como las condiciones asociadas a la enfermedad y sus tratamientos ${ }^{(1)}$.

Atendiendo a la bibliografía, podemos encontrarnos cifras que oscilan entre un $12 \%$ y $47 \%$ de los pacientes con cáncer cumplen criterios de trastorno mental. Dichas diferencias dependen de las muestras de pacientes analizados ${ }^{(2)}$.

En población española, Gil et al. encontraron en una muestra de 400 pacientes oncológicos españoles que la prevalencia de trastornos psicopatológicos en estos pacientes ronda el $24 \%$ de la población, siendo el trastorno adaptativo el más habitual $(77,3 \%)$. Le sigue la distimia $(5,2 \%)$, el trastorno depresivo mayor $(4,1 \%)$ y el primer trastorno de ansiedad que surge en la tabla es el de fobia específica $(3,1 \%)$. Al igual que en población anglo-americana, las fobias es el trastorno de ansiedad más común en pacientes oncológicos ${ }^{2}$

Según el Dsm IV ${ }^{(3)}$, una fobia es un temor acusado y persistente que es excesivo e irracional, desencadenado por la presencia o anticipación de un objeto o situación específicos. Una reacción a un miedo fó- bico se caracteriza por desatarse ante la presencia o la anticipación de un estímulo o situación concreta, desarrollándose un conjunto de reacciones fisiológicas, cognitivas o motoras ${ }^{(3)}$. Dentro de pacientes oncológicos, es más habitual encontrar literatura acerca de pacientes con fobia a la quimioterapia, a la sangre, a inyecciones, a espacios cerrados,... Fobias más comunes dentro de la psicooncología y de la población clínica para los que incluso se han realizado protocolos de exposición con el fin de superar dicho miedo. Pero dentro de las fobias específicas, nos podemos encontrar con fobias a ciertos aspectos menos comunes. Tal es el caso que se va a presentar, en el que la paciente, mujer afectada de mama, contrajo una fobia a padecer linfedema, lo que le llevó a negarse a realizar cualquier movimiento o esfuerzo con el brazo en el que se le realizó la mastectomía.

\section{PRESENTACIÓN DEL CASO}

Mujer de 38 años, en proceso de separación y con dos hijos de 17 y 11 años, que viven con ella.

\section{ASPECTOS CLÍNICOS}

Estudiada a la edad de 37 años por presentar un pequeño bulto en la mama derecha con crecimiento progresivo. En la mamografía se objetivó una asimetría de novo en la mama derecha de unos $4 \mathrm{~cm}$. La anatomía patológica reveló un carcinoma ductal infiltrante con receptores de estrógenos y de progesterona positivos (+++), Her2 negativo, ki67 (índice de proliferación) $25 \%$. En el Comité de tumores se decide quimioterapia neoadyuvante (preoperatoria). Recibió 4 ciclos de AC (Adriamicina y Ciclofosfamida) y 12 de Taxol semanal, con buena respuesta clínica. Intervenida (21/1/10) mediante mastectomía derecha y biopsia de ganglio centinela, que resultó 
positivo, por lo que se continuó con linfadenectomía axilar. La anatomía patológica definitiva evidenció un carcinoma ductal infiltrante de al menos $6 \mathrm{~cm}$, grado 3 , con invasión perineural; el borde superior de la pieza extirpada estaba afectada por tumor, el borde profundo muy próximo; receptores de estrógenos y de progesterona positivos (+++), Ki67 25\%, Her2 negativo; 2 ganglios afectos de los 15 extirpados; 1 ganglio axilar intramamario negativo. Reintervenida (3/3/10) con ampliación de márgenes de la mastectomía derecha, realizando una resección amplia de piel y tejido glándulo-graso de cuadrantes supero externos incluyendo fascia muscular; exéresis-biopsia de tumoración axilar derecha adherida a vena axilar; cobertura del defecto con colgajo de dorsal ancho basado en la rama descendente de la arteria toraco-dorsal. La anatomía patológica no mostró presencia de malignidad. Posteriormente recibió radioterapia complementaria y Tamoxifeno.

Siguiendo el protocolo de actuación con las mujeres afectadas de mama de la asociación española contra el cáncer en Cáceres, se visita a la paciente recién operada para valorar su estado emocional, e informar sobre el linfedema y las prótesis mamarias. Durante esta entrevista, la paciente refiere que su marido la ha abandonado durante el proceso de quimioterapia, pero que se encuentra muy bien y que no necesita ayuda de nadie. Rechaza la continuidad de la entrevista porque hay familiares esperándola y comenta que ya leerá el libro sobre información del linfedema. La psicooncóloga recoge en el historial su propia impresión de una actitud extremadamente alegre y positiva por parte de la paciente, teniendo en cuenta la situación que la paciente había informado y su rechazo a continuar la entrevista.

En Junio del 2010 acude a consulta remitida por su oncóloga, ya que presenta un gran miedo a realizar cualquier movi- miento con el brazo derecho por temor a contraer el linfedema. En ese momento, la paciente ha finalizado las sesiones de radioterapia y se encuentra en proceso de revisión.

La paciente se muestra colaboradora y receptiva, siendo consciente de que presenta un miedo exagerado a contraer el linfedema, pero que le es imposible de superar.

Los problemas que se detectan en esta primera valoración son:

- Miedo exacerbado a contraer el linfedema, lo que le lleva a no realizar ninguna actividad con el brazo derecho. No coge ningún objeto con ese brazo ni lo mueve.

- Información inadecuada sobre el linfedema, qué es y cuál es el modo de prevenirlo.

- Tristeza y deseos de llorar, pero le resulta imposible

- Bloqueo emocional ante el abandono de su esposo.

- Negación al hablar del proceso de separación

- Preocupación por sus hijos, que no desean tener relación con su padre.

- Sentimientos de culpa por la situación de la familia propia.

Objetivos de la paciente:

- Vencer el miedo a mover el brazo.

- Mejorar la relación de sus hijos con su padre.

\section{EVALUACIÓN}

- Entrevista semiestructurada siguiendo el formato de historia clínica de la asociación española contra el cáncer y observación directa.

- Se realizó valoración psicométrica a través del Cuestionario de Ansiedad-Estado-Rasgo (STAI); el inventario de depresión de BECK (BDI) y el cuestionario de calidad de vida para mujeres de mama (FACT-B +4) 
- La aplicación del STAI arrojó una puntuación directa en ansiedad estado de 27, situando a la paciente en el centil 65. La puntuación directa en ansiedad rasgo es de 27 , colocando a la paciente en el centil $60^{(4)}$.

- La versión española del Inventario de depresión de Beck (BDI) sitúa a la paciente por enciman del punto de corte para graduar el síndrome depresivo leve (Puntuación de 11)(5).

- El cuestionario de calidad de vida FACT-B +4 para mujeres afectadas de mama arroja una puntuación global de 104 sobre 146, lo que denota que la calidad de vida de la paciente se ha visto mermada debido al proceso oncológico que está padeciendo. Las puntuaciones por subescalas fueron las siguientes: Estado Físico (EF) 27 sobre 28; Ambiente familiar y social (AFS) 18 sobre 28; Estado emocional (EE) 17 sobre 24; Capacidad de funcionamiento personal (CFP) 14 sobre 28 y otras preocupaciones (OP) 28 sobre $36^{(6)}$.

- La evaluación psicométrica y la entrevista semi estructurada corroboran que la paciente padece una fobia específica a contraer linfedema, así como un gran bloqueo emocional, en base a lo cual se estructura la terapia.

\section{ANÁLISIS FUNCIONAL DEL PROBLEMA}

Es necesario analizar los problemas de la paciente desde tres perspectivas: Fisiológica, cognitiva y motora ${ }^{(7)}$.

A nivel fisiológico, cabe destacar la gran intervención que se le ha realizado, ya que tras la mastectomía con linfadenectomia de Enero del 2010, tuvo que ser reintervenida en Marzo de ese mismo año, ampliando los márgenes de la mastectomía, llegando a la fascia muscular.
A nivel motor, la paciente se niega a realizar cualquier actividad con el brazo, por muy mínimo que sea. Esto ha llevado a que no realice actividades cotidianas y a que necesite ayuda para actividades básicas de la vida diaria (Vestirse, asearse, comer,...) Cualquier mínimo movimiento con el brazo supone una situación de alta ansiedad para ella.

Suele consultar en Internet las dudas que se le presentan y ha visualizado imágenes de linfedemas irreversibles y de elefantiasis, lo que retroalimenta su conducta de no mover el brazo para evitar un cuadro clínico similar en ella.

A nivel cognitivo, se detectan importantes concepciones erróneas sobre el linfedema, considerando que el brazo se le va a hinchar de un día para otro, generándose con cualquier tipo de movimiento, esfuerzo o toma de peso con el brazo.

Cabe destacar su hiperresponsabilidad y autoexigencia. Tiene muy interiorizado el sentimiento de culpa de la situación en la que está su familia: "Está todo mal por mi culpa y por esta dichosa enfermedad".

Una idea que aparece frecuentemente en su discurso que es ella quien se debe encargar de todo para que las cosas vayan bien. Aparecen alusiones tales como: "Yo tengo que estar bien", "Soy quien tira del carro".

Del mismo modo, considera que llorar o mostrar tristeza supone una muestra de flaqueza y generar preocupación innecesaria a sus familiares: "No me puedo permitir perder el tiempo en lloriqueos".

Por último, la paciente muestra un gran bloqueo de emociones y actitud de alerta hacia cualquier tema comprometido que le haga venirse abajo, especialmente el proceso de divorcio de su esposo, con lo que muestra gran indiferencia e intenta no hablar de ello. 


\section{Determinantes}

Como antecedentes externos remotos, cabe destacar el hecho de que la paciente siempre ha sido quien se ha hecho responsable de situaciones no acordes a su edad. Al ser la mayor de una familia numerosa, se ha encargado del cuidado de sus hermanos menores. Del mismo modo, fue madre muy joven (con 20 años), lo que le ha llevado a ser siempre un punto de apoyo y referencia para toda su familia.

Su ex-marido ha padecido problemas psiquiátricos desde la juventud, con trastorno depresivo recidivante, episodios que ha vivido con mucha angustia. Debido a la enfermedad de su esposo, la paciente ha sido siempre la persona en que él y sus hijos se han apoyado.

También hemos de tener en cuenta el diagnóstico de cáncer de mama y todo el proceso de tratamiento por el que ha pasado.

Como antecedentes internos remotos, destaca su alto nivel de auto exigencia, su gran sentido de la responsabilidad hacia a todos y su gran autocontrol para evitar mostrar sus sentimientos. No hemos de olvidar su rechazo a la atención psicológica y a que se le orientara debidamente sobre el linfedema y el modo de prevenirlo.

Con respecto a los antecedentes externos recientes, podemos señalar la decisión de su marido de divorciarse de ella. Debido a los efectos del tratamiento, los roles en la familia cambiaron, encontrándose su esposo (con trastorno depresivo recidivante) por primera vez con la necesidad de tomar un rol de cuidador, no de enfermo. Este hecho le llevó a abandonar el domicilio familiar y volver a la casa materna, donde volvió a adquirir el rol dependiente y pasivo que ha tenido siempre. El motivo por el que alega divorciarse es porque teme perderla si fallece, aunque todo apunta el hecho de verse incapaz de asumir los cambios que se están produciendo en el núcleo familiar; así como los cambios que se han producido en su protocolo de tratamiento, al tener que realizársele una intervención quirúrgica más agresiva.

Como antecedentes internos recientes, nos encontramos sus pensamientos de culpa por los que considera que la situación familiar se debe por ella y su idea distorsionada del linfedema, Ilevándole a evitar cualquier tipo de actividad con el brazo.

\section{Consecuentes}

A corto plazo, el evitar cualquier situación que suponga el movimiento o esfuerzo del brazo hace que disminuya su nivel de ansiedad de manera temporal mediante un mecanismo de refuerzo negativo.

Sus auto imposiciones de tener que estar bien y positiva, así como la evitación de hablar sobre su exmarido, refuerzan su bloqueo emocional y cualquier situación que pueda favorecer la expresión de sentimientos y de muestra de debilidad.

El mantenimiento de estas circunstancias puede generar a medio y largo plazo un aislamiento social y una situación de dependencia de la paciente hacia los demás, ante la negativa de realizar cualquier ejercicio con el brazo, así como el afianzamiento de una fobia específica a contraer linfedema.

Esta dependencia puede favorecer los sentimientos de culpa e inutilidad, que unidos a la gran hipervigilancia de la paciente para controlar sus emociones, favorezca la aparición de otros cuadros psicopatológicos, así como una sensación de aislamiento y soledad por parte de la paciente.

\section{Recursos de la paciente}

A pesar de la gran ansiedad que le supone mover el brazo, es consciente de que no está teniendo una reacción normal y se muestra colaboradora y dispuesta a solu- 
cionar el problema. En ello le va a ayudar su espíritu de lucha, constancia y ganas de superación. Cuenta con un gran apoyo familiar, tanto de su familia de origen como de sus hijos, que la apoyan en todo momento. Socialmente, es una persona muy popular en su municipio de origen y el hecho de que su marido la abandonara en pleno tratamiento de quimioterapia ha supuesto que la apoyen aún más y que la acojan.

A pesar de poseer un nivel cultural medio, la paciente presenta un nivel intelectual alto, lo que ayuda mucho a la hora de clarificar ideas. Su autoimagen corporal es buena, sin que le haya generado grandes problemas las secuelas de la mastectomía.

\section{Diagnóstico DSM-IV}

Eje I: Fobia específica a contraer linfedema (F40.2)

Eje II: Diagnóstico ausente.

Eje III: Carcinoma de mama ductal infiltrante.

Eje IV: Problemas relativos al grupo primario y de apoyo: Divorcio de su marido

Problemas laborales: Imposibilidad de realización de su profesión debido a secuelas del tratamiento.

Eje V: E.E.A.G: $60 \%$ (En momento de consulta)

\section{ANÁLISIS DEL CASO}

En un muy corto espacio de tiempo, la paciente ha sufrido grandes en cambios en su vida, debido al diagnóstico de cáncer de mama y al tratamiento derivado de él para su cura. Dicha enfermedad ha supuesto un cambio de rol, ya que la paciente ha sido siempre la cabeza de la familia, quien se ha encargado de todo. Los tratamientos y sus secuelas han obligado a un cambio familiar que ha trastocado a todos los miembros de la familia, especialmente a su marido, enfermo psi- quiátrico crónico, quien decidió abandonar a su esposa durante el tratamiento por temor, según refiere la paciente "A perderla si muere".

El hecho del cáncer y de la separación ha supuesto un gran sufrimiento para la paciente, pero debido a su gran hiperresponsabilidad y autoexigencia, derivado de su papel de matriarca, ha hecho que la paciente evite todo aquello que supone punto de flaqueza en ella:

- Las secuelas de su tratamiento: Negación de que los ciclos generan malestar en ella y cuidados derivados de la mastectomía con linfadenectomía, ya que es un indicador de vulnerabilidad.

- Evitación total y actitud indiferente ante el abandono y divorcio de su marido.

- Rechazar inicialmente la atención psicológica en el momento de crisis (El abandono de su esposo y la operación de la mama se solaparon en el tiempo), por la idea distorsionada de lo que supone una asistencia psicológica, ya que consideraba que asistir a terapia suponía ser débil emocionalmente.

- Tomar una actitud autodidacta ante ciertos aspectos de su enfermedad, considerando que podía afrontarlos ella sola.

Esta actitud evitativa ha desembocado en:

1. Rechazar la información adecuada por parte de un profesional sobre la prevención del linfedema, auto-informándose sobre todo consultando en la web, viendo fotos de personas con linfedema irreversible y generando una idea distorsionada sobre el dicha dolencia, conduciéndole a una fobia a contraer dicha dolencia, negándose a realizar cualquier movimiento con el brazo. 


\section{Tabla 1. Modelo formulación del caso}

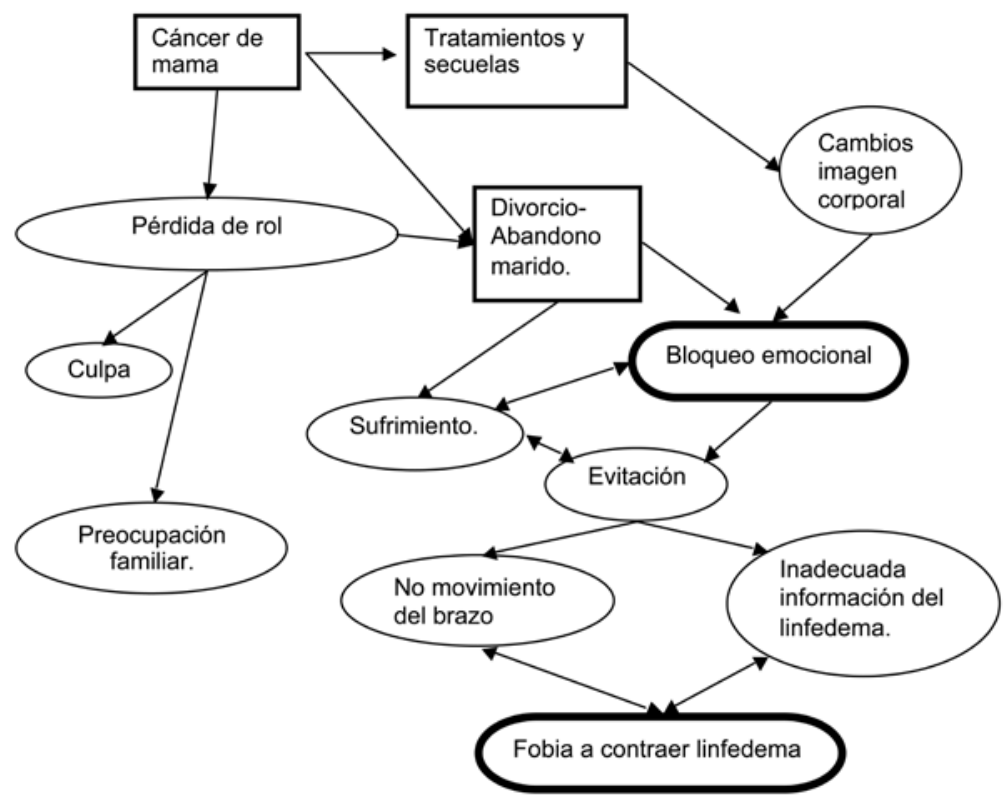

2. Su deber de cabeza visible de la familia le genera pensamientos de culpa ante la situación de divorcio, por lo que emocionalmente se ha bloqueado totalmente, fingiendo normalidad y control y así evitar la pérdida de rol de matriarca.

3. Otro problema desemboca de estas dos actitudes, ya que el hecho de no mover el brazo bajo ningún concepto genera una gran dependencia para con los demás y una gran preocupación familiar, justamente lo que la paciente intenta evitar con ese bloqueo emocional y empeño de estar bien, por lo que esos pensamientos de culpa aumentan.

\section{OBJETIVOS}

- Conseguir que la paciente supere el miedo a poder mover el brazo y al linfedema.

- Realizar una adecuada psico-educación sobre el modo de prevenir el linfedema.
- Disminuir su ansiedad.

- Reestructuración cognitiva sobre las emociones y el hecho de poder permitirse expresarlas, así como sentimientos de culpa.

- Desbloquear emocionalmente a la paciente.

- Superación del abandono y divorcio de su marido.

\section{PLAN DE TRATAMIENTO}

A continuación se describe en contenido de cada una de las sesiones, con un breve comentario de las actividades concretas realizadas y el desarrollo de las mismas:

\section{Sesión 1:}

Evaluación: entrevista semiestructurada según el formato de historia clínica para pacientes de la asociación española contra el cáncer y aplicación de cuestionarios (BDI, STAI y FACT-B +4). 
Explicación del plan de tratamiento: explicación del método de exposición ${ }^{(8)}$ para superación de fobias específicas. tica $^{(9)}$.

Explicación de Respiración diafragmá-

Tareas para casa: realización de jerarquía de situaciones que le resultaran muy ansiosas y entrenamiento de la respiración diafragmática.

Cabe destacar que la paciente, al tener que realizar los cuestionarios, se negaba a coger el bolígrafo con la mano derecha (brazo afectado, a pesar de que ella es diestra). Con ayuda y su esfuerzo, terminó realizando todos los cuestionarios con dicha mano, algo que le supuso de gran refuerzo.

\section{Sesión 2:}

Psicoeducación: explicación correcta y adecuada sobre el linfedema, cómo su puede generar y qué se puede hacer para prevenirlo: Ejercicios de rehabilitación y cuidados con el brazo ${ }^{(10)}$.

Revisión de jerarquía de situaciones ansiógenas: se repasa la lista para asegurar que la jerarquía está en el orden adecuado.

Explicación de la autoexposición: se explica los registros que se van a realizar y cómo ha de rellenarlos cuando realice las tareas ella sola.

Repaso de la respiración diafragmática e introducción de relajación por visualización ${ }^{(9)}$ : se descarta la relajación muscular progresiva porque la paciente mantiene agarrotados el brazo, hombros y zona derecha del cuerpo, por lo que nos decantamos por la relajación por visualización para evitar un empeoramiento en esa zona.

Tareas para casa: afianzar la jerarquía y realizar ejercicios de respiración diafragmática y de relajación.

Cabe destacar que la paciente, confiada por el hecho de escribir con la mano derecha, ha hecho por su cuenta algunos avances, como coger la esponja en el baño y la cuchara del café.

\section{Sesión 3:}

Psicoeducación: repaso sobre qué es el linfedema y cómo se puede prevenir, para evitar errores.

Estabilización de la jerarquía: la lista la compone las siguientes actividades, de menor a mayor ansiedad: Comer, vestirse, actividades de limpieza de la casa, aseo personal y realización de actividad física.

Hemos de recordar que la paciente tiende a minimizar todo lo que le sucede, por lo que no puntuó ninguna situación con cifras de 100, aunque probablemente sí que experimentara niveles de ansiedad mayores a los referidos.

Al realizar la jerarquía, se comprobó que existían ítems que podían ser introducidos en una temática global (ej. Limpiarme con la esponja y secarme con la toalla se introdujeron en el ítem global de Aseo personal), por lo que los registro incluían dichas actividades, de tal manera que la paciente iba simultaneando tareas si así

\section{Tabla 2. Jerarquía de la exposición}

\begin{tabular}{|c|c|}
\hline TAREA & NIVEL DE ANSIEDAD \\
\hline Comer & 40 \\
\hline Vestirse & 60 \\
\hline Actividades de limpieza de la casa & 70 \\
\hline Aseo personal & 80 \\
\hline Ejercicio físico & 90 \\
\hline
\end{tabular}




\begin{tabular}{|c|c|c|c|c|}
\hline 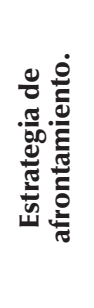 & 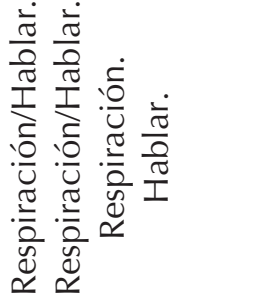 & 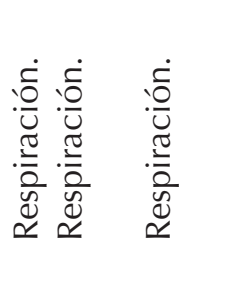 & 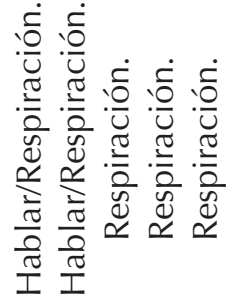 & 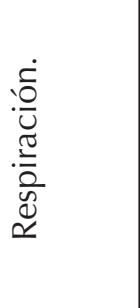 \\
\hline 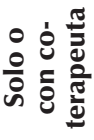 & 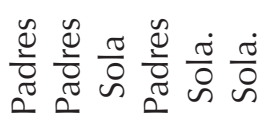 & 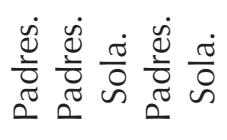 & 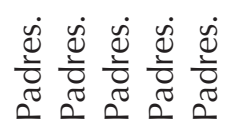 & 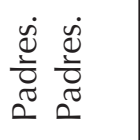 \\
\hline 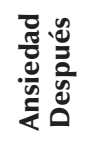 & 0 an 000 & $\stackrel{\sim}{-} \underset{\sim}{-1} \wedge \sim$ & 위유 는 응 & $\stackrel{0}{-} \ln 0$ \\
\hline 宽 & 으ำ เก ำ ำ ㅇำ & $\hat{b} \stackrel{\circ}{\circ} \stackrel{m}{\sim} \stackrel{\infty}{-}$ & 용ํํ $\stackrel{m}{\forall}$ & 윤은 \\
\hline 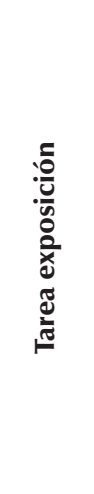 & 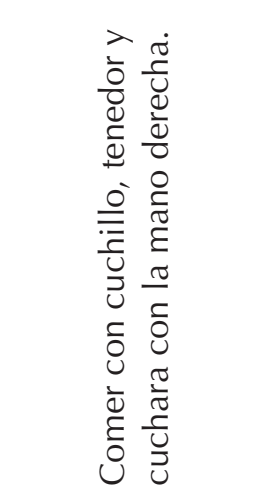 & 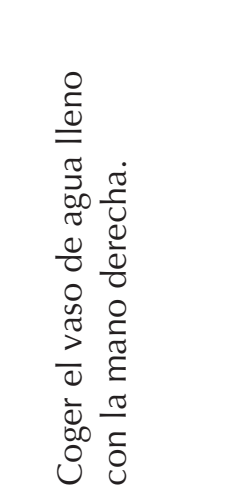 & 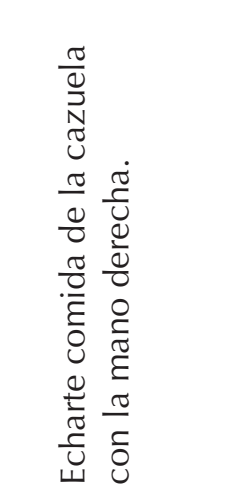 & 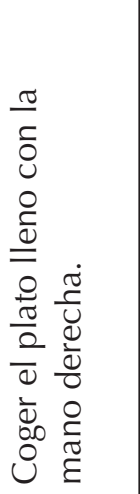 \\
\hline 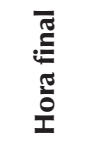 & 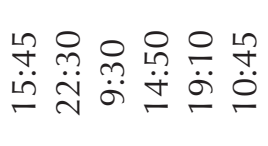 & 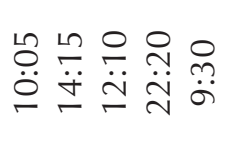 & 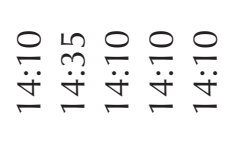 & 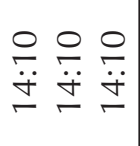 \\
\hline 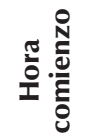 & 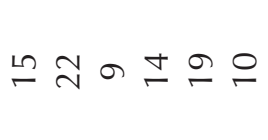 & $\stackrel{ }{-} \underset{\sim}{ }$ & 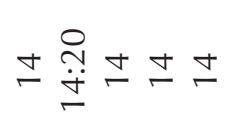 & $\stackrel{ナ}{ナ}$ \\
\hline 胥 & 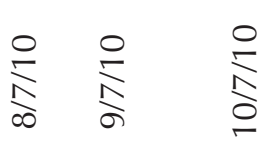 & 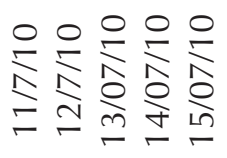 & 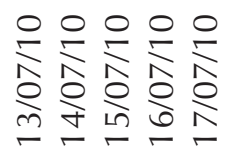 & 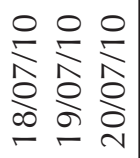 \\
\hline
\end{tabular}


se sentía capaz, tal y como se puede ver el registro.

Tareas para casa: según de acuerdo con la paciente, teniendo en cuenta que vive en otro municipio y tiene dificultades para trasladarse a la ciudad, se envía para casa dos actividades: Comer y vestirse (ponerse el pantalón y abrocharse con las dos manos; ponerse y quitarse una camiseta de tirantes con las dos manos; ponerse una camisa, empezando por la mano izquierda (brazo no afectado) y ponerse una camisa empezando por la mano izquierda (realizando así mayor giro con el brazo derecho) y abrocharse con las dos manos).

\section{Sesión 4:}

Revisión de tareas: ha podido realizar todas las tareas, encontrando más dificultad a la hora de coger el vaso del agua, pero que está superado.

Tareas para casa: registro de actividades de limpieza de la casa (limpiar el polvo con la mano derecha, barrer y fregar con las dos manos; planchar con la mano derecha, hacer los cuartos de baño con la mano derecha).

La paciente se muestra muy motivada y contenta por los progresos que va consiguiendo, lo que favorece la adherencia a la terapia. Comenta que se encuentra "mitad y mitad y media de lo que soy yo". Gana confianza con la terapeuta.

\section{Sesión 5:}

Revisión de tareas: superada sin dificultad las actividades de limpieza de la casa.

Tareas para casa: registro de aseo personal (limpiarme con la esponja con la mano derecha; secarme con la toalla y con las dos manos; pintarme con la mano derecha; depilarme las axilas, lavarme el pelo). Recordatorio de que debe seguir realizando las otras actividades. La paciente co- menta que no ha dejado de hacerlas, una vez superadas.

La paciente comenta a expresar qué ha supuesto para ella toda la enfermedad, encontrándose muy cansada anímicamente con el sobre esfuerzo que ha realizado para parecer que estaba muy bien. Aprovechando el voto de confianza que realiza, se permite ventilación emocional y se comienza a realizar reestructuración cognitiva sobre las emociones y el hecho de tener que estar siempre bien. Se compara su situación a la de un familiar (con historia similar) dándose cuenta por sí misma el alto nivel de autoexigencia y de perfeccionismo que se autoimpone, generándole gran angustia y malestar.

\section{Sesión 6:}

Revisión de tareas: superada sin dificultad las actividades de aseo personal.

Ventilación emocional: la paciente comenta que por fin ha llorado y que le ha venido muy bien, pero le preocupa que sus hijos sobretodo la vean llorar y mal. Comienza a hablar sobre su divorcio, apareciendo esos sentimientos de culpa porque cree que pudo haber dejado a un lado a su marido debido a la enfermedad, que no lo atendió como debía. Se realiza reestructuración cognitiva con la técnica de descubrimiento guiado para dichos imperativos y se recuerda lo trabajado en la sesión anterior con respecto a su nivel de exigencia.

Explicación de programación de quejas: aunque esta técnica tiene como fin un cambio cognitivo, se utilizó en este caso como "hora basura"(11), un tiempo en que la paciente pudiera llorar y desahogarse sin temor a que sus hijos la vieran, algo que le resultaba muy difícil, y poder permitirse con su actividad diaria a lo largo del día.

Tareas para casa: registro de actividad física (Correr mínimo 10 minutos; hacer 
abdominales (20-25 como mínimo); hacer "pesas" con caja de leche; flexiones (5 mínimo) con rodillas cruzadas). Usar su "Hora basura" todos los días.

\section{Sesión 7:}

Revisión de tareas: superado sin dificultad las actividades de actividad física.

Repaso de tareas de exposición: la paciente ha superado totalmente su miedo a mover el brazo, realizando una vida totalmente normal. Posee una información real y ajustada de lo que puede realizar con el brazo, cuidándolo de una manera adecuada.

Proceso de divorcio: la paciente comenta los sentimientos que le genera su ex marido y la necesidad que tiene de querer decirle muchas cosas, pero él se niega a hablar con ella. Tratamos el hecho de no poder obligar a una persona si no quiere a hablar, pero que ella también necesita desahogarse.

Tareas para casa: carta de despedida para su exmarido, a modo de cierre del proceso.

\section{Sesión 8:}

Proceso de divorcio: escribir la carta le ha liberado. Comenta que sus sentimientos de rabia y de culpa han pasado a sentir lástima por él, ya que se encuentra solo y sus hijos no quieren pasar mucho tiempo con él.

Comunicación con los hijos: a la paciente le preocupa la mala relación entre sus hijos y su padre y busca el modo de mejorarla. Ahondando en el problema, la paciente ha intentado incluso mantener una relación cordial con su exmarido, algo a lo que él se ha negado. Se trabaja con la paciente la adjudicación de responsabilidades ante el hecho de la relación padrehijos, haciéndola ver que ella no puede solucionar todo, que es algo que depende de ellos también.
Tareas para casa: hablar con sus hijos respecto al tema.

\section{Sesión 9:}

Comunicación con los hijos: al hablar con ellos sobre la relación con su padre, el hijo mayor (Ya de 18 años) le comenta que es su decisión no hablar con su padre y que es algo entre ellos dos. El hijo menor comenta que con quien está realmente es con su abuela paterna cuando va a casa de su padre, ya que el padre casi nunca está. Se vuelve a trabajar límites con la paciente, haciéndola ver que no es responsabilidad suya.

Por lo demás, presenta una vida totalmente normal. Tras 9 sesiones de tratamiento de una duración de 1 hora cada una aproximadamente, se concierta sesión de seguimiento al mes, que posteriormente se irá espaciando dependiendo de la evolución.

\section{RESULTADOS OBTENIDOS}

El objetivo más importante de la terapia era erradicar la fobia que la paciente había contraído a padecer linfedema. La paciente superó todas las tareas de la exposición, pudiendo realizar una vida totalmente normal, con una idea adecuada de qué es el linfedema y de cómo se puede prevenir.

Los niveles subjetivos de ansiedad y sintomatología depresiva se encontraban en niveles de normalizar el tratamiento psicológico, algo que corrobora la valoración psicométrica, de la que se obtuvo los siguientes resultados:

- BDI: 6, lo que indica ausencia de sintomatología depresiva.

- STAI: Ansiedad estado con una puntuación directa de 19, situando a la paciente en el centil 45. Ansiedad rasgo con una puntuación directa de 19, llevando a la paciente al centil 30 . 
- Fact-B +4: Puntuación de 104 sobre 146, por lo que su calidad de vida sigue siendo la misma.

Actualmente, la paciente sigue realizando una vida totalmente normal. Tiene una actividad diaria elevada y se encuentra tranquila y contenta consigo misma. La relación con su ex pareja es cordial y la relación con su familia ha cambiado, poniendo límites de tal modo que no se vea sobrecargada con los problemas de los demás, permitiéndose a su vez mostrar sus emociones sin temor alguno.

\section{CONCLUSIONES}

El trastorno fóbico se solucionó sin problemas siguiendo el tratamiento de exposición, corroborando así que esta técnica es la más adecuada para superar problemas fóbicos en pacientes oncológicos.

Teniendo en cuenta que el linfedema es la secuela más importante del tratamiento del cáncer de mama 1 de cada 4 mujeres desarrollará esta complicación) ${ }^{(8)}$. Cabe señalar la gran importancia de realizar un buen trabajo de psicoeducación sobre el linfedema y sus consecuencias, así como el modo de prevenirlo en mujeres intervenidas de linfadenectomía disminuyendo así la probabilidad de que la paciente desarrolle problemas psicopatológicos posteriores.

\section{REFERENCIAS BIBLIOGRÁFICAS}

1. Ballesteros M, Cruzado JA, Lozano A, Sánchez P. Evaluación y tratamiento psicológico de un caso con cáncer de colon con fobia a la quimioterapia y trastorno adaptativo. Psicooncología 2005;2(1):139-48.

2. Gil FL, Costa G, Pérez FJ, Salamero M, Sánchez N, Sirgo A. Adaptación psicoló- gica y prevalencia de trastornos mentales en pacientes con cáncer. Med Clin (Barc). 2008;130(3):90-2.

3. American Psychiatric Association(APA). Manual Diagnóstico y Estadístico de los Trastornos Mentales (DSM-IV). Barcelona: Masson; 1995.

4. Spielberger CD, Gorsuch RL, Lushene RE. Cuestionario de Ansiedad Estado-Rasgo (STAI) Madrid: TEA; 1997.

5. Vázquez C, Sanz J. Fiabilidad y valores normativos de la versión española del Inventario para la depresión de Beck de 1978. Clin Salud 1997;8:403-22.

6. Belmonte R, Garin O, Segura M, Sanz J, Marco E, Ferrer M. Validación de la versión española del Functional Assessment of cancer Therapy Questionnaire for breast cancer (FACT-B+4). Med Clin (Barc) 2011;137:685-8.

7. Barraza A. Evaluación e intervención psicológica en un caso de trastorno adaptativo mixto con ansiedad y estado de ánimo depresivo en una mujer con recidiva por cáncer de mama. Psicooncología 2004;1(1):165-78

8. Echeburúa E., De corral, P. Técnicas de exposición: variantes y aplicaciones. En Labrador FJ, Cruzado JA, Munoz M, editores. Manual de Modificación de Conducta. Madrid: Pirámide; 1993.p. 422-56.

9. Labrador FJ, De la Puente ML, Crespo M. Técnicas de control de la activación: Relajación y respiración. Manual de modificación de conducta. Madrid: Pirámide; 1993.p.367-95.

10. Asociación Española Contra el Cáncer. Linfedema, prevención y tratamiento. Madrid 2010.

11. Muñoz M, Vázquez C. Procedimientos de intervención cognitiva. Técnicas cognitivas de intervención clínica. Madrid: Síntesis;2003 p. 91-145. 